\title{
Solvothermal synthesis of $\mathrm{CdO}$ and $\mathrm{CuO}$ nanocrystals
}

\author{
Moumita Ghosh ${ }^{\text {a,b }}$, C. N. R. Rao ${ }^{\text {a,b,* }}$ \\ a Solid State and Structural Chemistry Unit \\ Indian Institute of Science \\ Bangalore 560 -012 India \\ $b$ Chemistry and Physics of Materials Unit \\ and CSIR Centre of Excellence in Chemistry \\ Jawaharlal Nehru Centre for Advanced Scientific Research \\ Jakkur P.O., Bangalore 560064 India
}

\begin{abstract}
Nanocrystals of $\mathrm{CdO}$ have been obtained by the decomposition of the cupferron complex in the presence of tri-n-octylphosphine oxide (TOPO) under solvothermal conditions. The precursor : TOPO ratio plays an important role in determining the size of the nanocrystals. The nanocrystals have been characterized by electron microscopy, absorption spectroscopy and fluorescence spectroscopy, besides x-ray diffraction. The $\mathrm{CdO}$ nanocrystals are single crystalline and show evidence for quantum confinement. $\mathrm{CuO}$ nanocrystals could also be prepared by the decomposition of the cupferronate under solvothermal conditions, the particle size being controlled by the initial precursor concentration.
\end{abstract}

*Corresponding author. FAX: (+91)80-23622760, Email: cnrrao@jncasr.ac.in 


\section{Introduction}

$\mathrm{CdO}$ is a direct band gap $(2.3 \mathrm{eV})$ semiconductor, with an indirect band gap of $1.36 \mathrm{eV}$. Due to its large linear refractive index $\left(\mathrm{n}_{0}=2.49\right)$, it is a promising candidate for optoelectronics applications and other applications, including solar cells [1,2], phototransistors [3], photo-diodes [4], transparent electrodes [5] and gas sensors [6]. Reduction in the dimensionality of such materials from the three dimensional bulk phase to the zerodimensional nanoparticles can lead to enhanced nonlinearity, determined by the quantum size effects and other mesoscopic effects. Because of these interesting possibilities, there has been some effort to prepare nanoparticles of $\mathrm{CdO}$. Wu et al. [7] prepared a nanometer-sized $\mathrm{CdO}$ organosol from an aqueous solution of $\mathrm{Cd}\left(\mathrm{NO}_{3}\right)_{2}$, a surfactant and toluene, while Liu et al. [8] synthesized CdO nanoneedles by chemical vapour deposition. $\mathrm{CdO}$ nanowires have been synthesized by decomposing $\mathrm{CdCO}_{3}$ in a $\mathrm{KNO}_{3}$ salt flux [9]. Zou et al. [10] have prepared $\mathrm{CdO}$ nanoparticles by the micro-emulsion method employing AOT reverse micelles. There is also a report of stearate-coated $\mathrm{CdO}$ nanoparticles of 5-10 nm size range, obtained by the micro-emulsion method starting from an aqueous solution of a cadmium salt and stearic acid in xylene [11]. We have developed a new method to prepare $\mathrm{CdO}$ nanoparticles, wherein a cadmium precursor compound, is decomposed under solvothermal $[12,13]$ conditions in presence of a capping agent. For this purpose, we have employed cadmium cupferronate, $\mathrm{Cd}\left(\mathrm{C}_{6} \mathrm{H}_{5} \mathrm{~N}_{2} \mathrm{O}_{2}\right)_{2}$ as the precursor, and obtained $\mathrm{CdO}$ nanoparticles of different sizes by using tri-n-octylphosphine oxide (TOPO) as the capping agent [14]. We have 
characterized the nanoparticles by X-ray diffraction, electron microscopy and electronic absorption and emission spectroscopies. We have also synthesized $\mathrm{CuO}$ nanocrystals by the decomposition of the cupferronate [15] under solvothermal conditions.

\section{Experimental}

The cupferron complex of $\mathrm{Cd}$ (II), $\mathrm{Cd}\left(\mathrm{C}_{6} \mathrm{H}_{5} \mathrm{~N}_{2} \mathrm{O}_{2}\right)_{2}$, was prepared by the reaction of cadmium acetate with cupferron ( $\mathrm{N}$-nitroso-N-phenylhydroxylamine, ammonium salt). In a typical reaction, $1.27 \mathrm{~g}$ of $\mathrm{AR}$ grade $\mathrm{Cd}\left(\mathrm{CH}_{3} \mathrm{COO}\right)_{2}, 2 \mathrm{H}_{2} \mathrm{O}$ was dissolved in $25 \mathrm{~cm}^{3}$ of water in a beaker. In another beaker, $1.5 \mathrm{~g}$ of cupferron was solubilised in $60 \mathrm{~cm}^{3}$ of water. The two solutions were cooled at $0{ }^{\circ} \mathrm{C}$ and the cupferron solution slowly added to the solution of $\mathrm{Cd}\left(\mathrm{CH}_{3} \mathrm{COO}\right)_{2}$ under vigorous stirring. The white precipitate obtained was collected, washed with $2.5 \% \mathrm{NH}_{3}$ solution followed by milli-q water, to remove the excess cupferron. The complex was characterized by chemical analysis was as follows: C, $35.77 \%$ found (calculated 37.27\%); H, $2.665 \%$ (2.59\%); N, $14.09 \%$ (14.49 \%). Thermogravimetric analysis of the as-prepared $\mathrm{Cd}(\operatorname{cup})_{2}$ in a nitrogen atmosphere, showed that there was sharp weight loss due to decomposition around $250{ }^{\circ} \mathrm{C}$.

In a typical reaction, $0.1 \mathrm{~g}(0.0258 \mathrm{mmol})$ of $\mathrm{Cd}(\mathrm{cup})_{2}$ and $0.1 \mathrm{~g}(0.254 \mathrm{mmol})$ of tri-n-octylphosphine oxide (TOPO) were taken in $48 \mathrm{~cm}^{3}$ toluene and sealed in a Teflonlined autoclave of $80 \mathrm{~cm}^{3}$ capacity. The mixture was heated at $240{ }^{\circ} \mathrm{C}$ for $150 \mathrm{~min}$. A dark reddish brown solid, insoluble in toluene was obtained as the product. It was washed with toluene for several times, followed by methanol, and dried at $40{ }^{\circ} \mathrm{C}$ for $2 \mathrm{~h}$. This preparation with a $\mathrm{Cd}(\mathrm{cup})_{2}: \mathrm{TOPO}$ ratio of $1: 1$ yielded one-dimensional structures of CdO. Different $\mathrm{Cd}(\mathrm{cup})_{2}$ :TOPO ratios were employed to obtain nanocrystals of different 
sizes. When the ratio was 1:2 and 1:5, insoluble orange-colored solids were obtained, and the latter ratio also yielded yellow dispersions in toluene. The orange solid contained relatively bigger nanocrystals (18-30 $\mathrm{nm}$ diameter), which was washed thoroughly with toluene for further characterization. Nanoparticles were precipitated out from the yellow organosol by the addition of methanol. The precipitated nanocrystals which could be redispersed in toluene had diameters in the 3-12 nm range. The size of the nanocrystals could be changed by varying the relative concentrations of the reactants. With a 1:5 ratio of $\mathrm{Cd}(\operatorname{cup})_{2}: \mathrm{TOPO}$, we were able to synthesize toluene-soluble $\mathrm{CdO}$ nanocrystals, without any insoluble fraction.

In addition to $\mathrm{CdO}$ nanocrystals, we have been able to prepare $\mathrm{CuO}$ nanocrystals by decomposing $\mathrm{Cu}(\mathrm{cup})_{2}$ in toluene under solvothermal conditions. We have carried out the reactions in the absence of any capping agent and obtained different sizes of nanocrystals by varing the $\mathrm{Cu}(\mathrm{cup})_{2}$ concentration. The reactions yielded a dark brown organosol which was stable in toluene and hexane. By the addition of n-octylamine to the organosol in toluene, the $\mathrm{CuO}$ nanocrystals could be solubulized completely, giving rise to transparent solutions. In a typical reaction to obtain $10.5 \mathrm{~nm}$ nanocrystals, $0.2 \mathrm{~g}$ $(0.58 \mathrm{mmol})$ of $\mathrm{Cu}(\mathrm{cup})_{2}$ was taken in $48 \mathrm{~cm}^{3}$ toluene (75\% filling fraction) and sealed in a Teflon-lined autoclave of $80 \mathrm{~cm}^{3}$ capacity. The autoclave was heated at $180{ }^{\circ} \mathrm{C}$ for $300 \mathrm{~min}$.

X-ray diffraction (XRD) patterns of the samples were recorded in the $\theta-2 \theta$ BraggBrentano geometry with a Siemens D5005 diffractometer using $\mathrm{Cu}-\mathrm{K} \alpha(\lambda=0.151418$ nm) radiation. For transmission electron microscope (TEM) studies, a solution or a dispersion of the sample in a suitable solvent was allowed to evaporate on a carbon- 
coated $\mathrm{Cu}$ grid. A JEOL (JEM3010) microscope with an accelerating voltage of $300 \mathrm{kV}$ was used to obtain TEM images. UV-Vis absorption spectra of nanocrystals in toluene were recorded using a Hitachi U3400 spectrometer. The reference used was toluene solution of TOPO. Photoluminescence spectra were recorded with a Perkin-Elmer model LS50B luminescence spectrometer.

\section{Results and discussion}

In Fig.1, we show the XRD patterns of the $\mathrm{CdO}$ nanocrystals prepared in the presence of TOPO. Pattern (a) is that of the insoluble orange coloured solid product obtained with a $\mathrm{Cd}(\mathrm{cup}) 2$ : TOPO ratio of 1:2. The pattern is characteristic of the rocksalt structure with $\mathrm{a}=4.695 \AA$ (JCPDS card no 05-0640), as established by Rietveld profile analysis [16]. We show the goodness of fit as well as the difference profile in Fig.1a. Based on the x-ray line broadening, the particle diameter was estimated to be 18 $\mathrm{nm}$. In Fig.1b, we show the XRD pattern of nanocrystals, which are smaller than $18 \mathrm{~nm}$. The X-ray line widths show them to be around $12 \mathrm{~nm}$ in diameter. In Fig.2a, we show the TEM image of the nanocrystals corresponding to the XRD pattern in Figurelb. The particle size distribution is shown as an inset. The average particle size from TEM appears to be around $9.5 \mathrm{~nm}$. These particles are single crystalline as revealed by the high

resolution electron microscope image in Fig.2b. The particles are spherical or elliptical in shape, not unlike those reported by Dong et al. [11]. The fringe spacing of $2.68 \AA$ observed in the high-resolution electron microscope image (HREM) corresponds to the separation between the $\{111\}$ lattice planes, whereas the $2.336 \AA$ spacing corresponds to the separation between the $\{200\}$ planes. 
In Fig.3 (a), we compare the electronic absorption spectra of the TOPO-capped CdO nanocrystals with diameters of (a) $18 \mathrm{~nm}$, (b) $9.5 \mathrm{~nm} \pm 2 \mathrm{~nm}$ and (c) $4.5 \mathrm{~nm} \pm 1.5$ $\mathrm{nm}$. The $18 \mathrm{~nm}$ nanocrystals show an absorption edge around $2.5 \mathrm{eV}$ [curve (a) in Fig.3 (a)]. Recall that the bulk sample with an absorption edge of $2.3 \mathrm{eV}$, show a broad maxima around $500 \mathrm{~nm}$. The $9.5 \mathrm{~nm}$ nanocrystals show an absorption edge of $\sim 2.8 \mathrm{eV}$ while the $4.5 \mathrm{~nm}$ nanocrystals show an absorption edge of $\sim 3.27 \mathrm{eV}$, as revealed by spectra (b) and (c) respectively in Fig. 3 (a). The large blue-shift relative to the bulk $\mathrm{CdO}$ observed with the $4.5 \mathrm{~nm}$ nanocrystals is attributed to quantum confinement. The bulk exciton Bohr radius of $\mathrm{CdO}$ is not available in the literature and it is therefore difficult to conclude as to which confinement regime the $\mathrm{CdO}$ nanocrystals belong.

Photoluminescence spectra of $\mathrm{CdO}$ have been reported by $\mathrm{Wu}$ et al. [7] who suggest that the band at $480 \mathrm{~nm}$ arises from the transition between the conduction and valence bands. They also report a band at $530 \mathrm{~nm}$ due to near band-gap radiative combination. In Fig.3 (b), we show the photoluminescence spectra of the $18 \mathrm{~nm}$ and 4.5 $\mathrm{nm}$ nanocrystals recorded at the excitation wavelengths of $430 \mathrm{~nm}, 480 \mathrm{~nm}$, and $520 \mathrm{~nm}$. The $18 \mathrm{~nm}$ nanocrystals exhibit bands around $540 \mathrm{~nm}, 560 \mathrm{~nm}$ and $590 \mathrm{~nm}$ respectively at excitation wavelengths of $430 \mathrm{~nm}, 480 \mathrm{~nm}$ and $520 \mathrm{~nm}$. The corresponding bands of the $4.5 \mathrm{~nm}$ nanocrystals occur at $495 \mathrm{~nm}, 540 \mathrm{~nm}$ and $570 \mathrm{~nm}$. We observe the maximum blue-shift in the case of the lowest wavelength emission band, which is considered due to transition between valence and the conduction bands.

In Fig. 4 we show the typical XRD patterns of $\mathrm{CuO}$ nanocrystals, obtained by varing the concentration of $\mathrm{Cu}$ (cup)2 in toluene. The patterns (a), (b) and (c) correspond to particle sizes of 10.5, 6 and $4 \mathrm{~nm}$ respectively, as obtained from the x-ray line 
broadenings. These $\mathrm{XRD}$ patterns give the monoclinic structure of $\mathrm{CuO}$ in agreement with the literature (JCPDS card no 45-0937). TEM image of CuO nanocrystals with an average diameter of $10 \mathrm{~nm}$ is shown in Fig.5 (a). The inset in this frame shows the particle size distribution. The particles are highly crystalline in nature, as revealed by the HREM image, shown in Fig.5 (b). The line spacing of $2.35 \AA$ corresponds to the separation between $\{111\}$ planes.

\section{Conclusion}

$\mathrm{CdO}$ and $\mathrm{CuO}$ nanocrystals have been prepared successfully by a solvothermal route involving the decomposition of the metal cupferronate in toluene medium. As most of the transitional metals and some non-transition metals form complexes with cupferron, this route can be readily employed to prepare nanocrystals of various metal oxides soluble in organic solvents. The $\mathrm{CdO}$ and $\mathrm{CuO}$ nanocrystals obtained by us are single crystalline in nature as revealed by electron microscopy. The $\mathrm{CdO}$ nanocrystals of $4.5 \mathrm{~nm}$ diameter show evidence for quantum confinement. 


\section{References}

[1] C. Sravani, K. T. R. Reddy, O. Md. Hussain, P. J. Reddy J. Solar. Energy. Soc. India, $1(1996) 6$.

[2] L. M. Su, N. Grote and F. Schmitt, Electron. Lett. 20 (1984) 716.

[3] R. Kondo, H. Okimura and Y. Sakai, Jpn. J. Appl. Phys. 10 (1971) 1547.

[4] F. A. Benko and F. P. Koffyberg, Solid State Commun. 57 (1986) 901.

[5] A. Shiori Jpn. Patent No. 7 (1997) 909.

[6] D. R. Lide (Ed.), "CRC Handbook of Chemistry and Physics", 77th edn. (CRC Press, Boca Raton, 1996/1997). 3/278, p.12/97.

[7] X. Wu, R. Wang, B. Zou, L. Wang, S. Liu, J. Xu, J. Mater. Res. 13 (1998) 604.

[8] X. Liu, C. Li, S. Han, J. Han, C. Zhou, Appl. Phys. Lett. 82 (2003) 1950.

[9] Y. Liu, C. Yin, W. Wang, Y. Zhan, G. Wang, J. Mater. Sci. Lett. 21 (2001) 137.

[10] B. S. Zou, V. V. Volkov and Z. L. Wang, Chem. Mater. 11 (1999) 3037.

[11] W. Dong and C. Zhu, Opt. Materials, 22 (2003) 227.

[12] M. Rajamathi, R. Seshadri, Curr. Opin. Solid State Mater. Sci. 6 (2002) 337.

[13] S. Thimmaiah, M. Rajamathi, N. Singh, P. Bera, F. C. Meldrum, N. Chandrasekhar, R. Seshadri, J. Mater. Chem. 11 (2001) 186.

[14] C. B. Murray, D. J. Norris, M. G. Bawendi, J. Am. Chem. Soc. 115 (1993) 8706.

[15] J. Rockenberger, E. C. Scher, A. P. Alivisatos, J. Am. Chem. Soc. 121 (1999) 11595.

[16] J. -F. Bérar, P. Garnier, NIST Spec. Publ. 846 (1992) 212. 


\section{Figure Captions}

Fig.1. Powder XRD patterns of TOPO-capped CdO nanocrystals of (a) $18 \mathrm{~nm}$ (b) $12 \mathrm{~nm}$ diameter. Rietveld fit is shown in (a) along with the difference profile. The vertical lines at the top of the figure are the expected peak positions.

Fig.2. (a) TEM image of TOPO-capped $\mathrm{CdO}$ nanocrystals of an average diameter of 9.5 nm. The XRD pattern of these particles is shown in Fig. 1(b). Inset shows the size distribution of the nanocrystals. (b) HREM images of CdO nanocrystals.

Fig.3. (a) Electronic absorption spectra of TOPO-capped CdO nanoparticles with an average diameters of (a) $18 \mathrm{~nm}$ (b) $9.5 \mathrm{~nm}$ and (c) $4.5 \mathrm{~nm}$. (b) Photoluminescence spectra of $18 \mathrm{~nm}$ and $4.5 \mathrm{~nm} \mathrm{CdO}$ nanocrystals at different excitation wavelengths. Curves 1, 2 and 3 are the photoluminescence spectra of the $4.5 \mathrm{~nm}$ nanocrystals at excitation wavelengths of $430 \mathrm{~nm}, 480 \mathrm{~nm}$ and $520 \mathrm{~nm}$ respectively, $1^{*}, 2^{*}$ and $3^{*}$ correspond to the $18 \mathrm{~nm}$ particles.

Fig.4. XRD patterns of $\mathrm{CuO}$ nanocrystals of (a) $10.5 \mathrm{~nm}$, (b) $6 \mathrm{~nm}$ and (c) $4 \mathrm{~nm}$ diameter. The vertical lines at the top are the expected peak positions.

Fig.5. (a) TEM image of $\mathrm{CuO}$ nanocrystals. Inset shows the size distribution of the nanocrystals. (b) HREM image of a single $\mathrm{CuO}$ nanocrystal. 


\section{Figure 1}

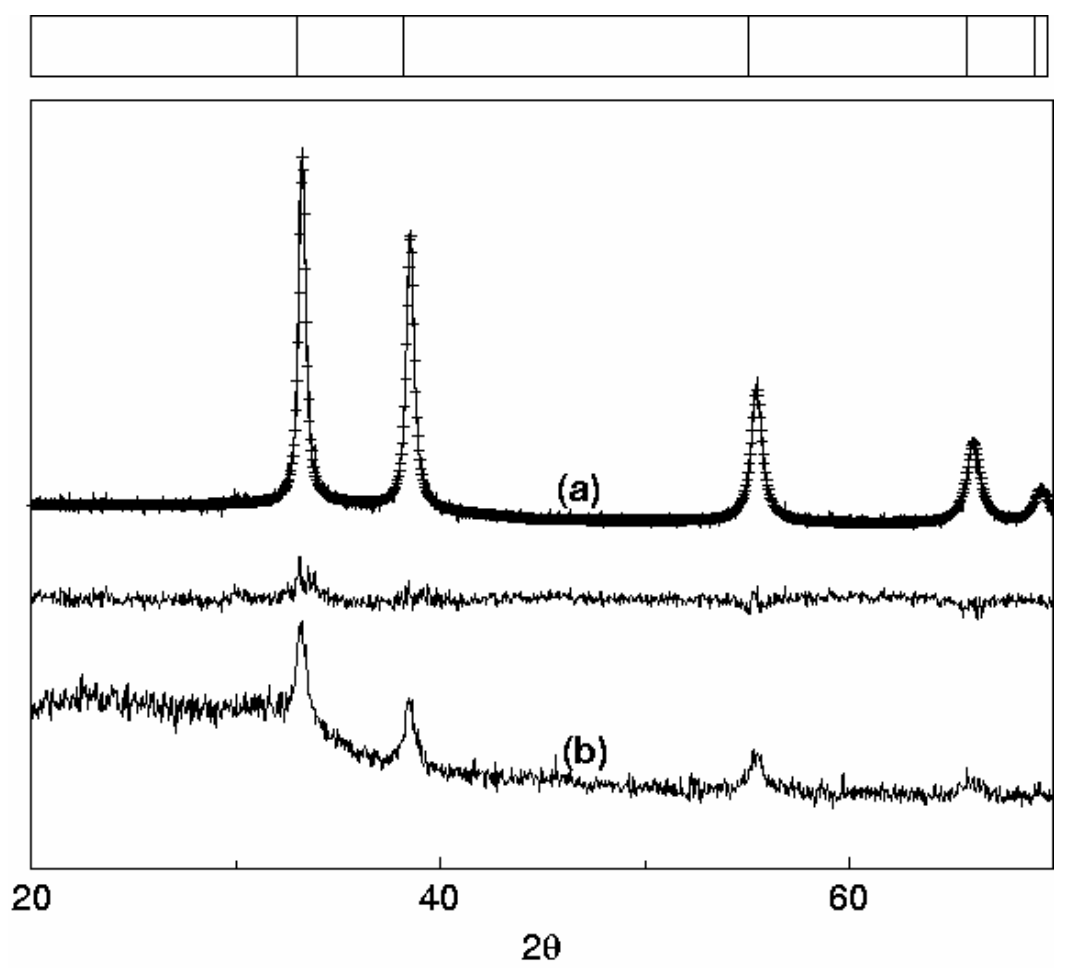


Figure 2
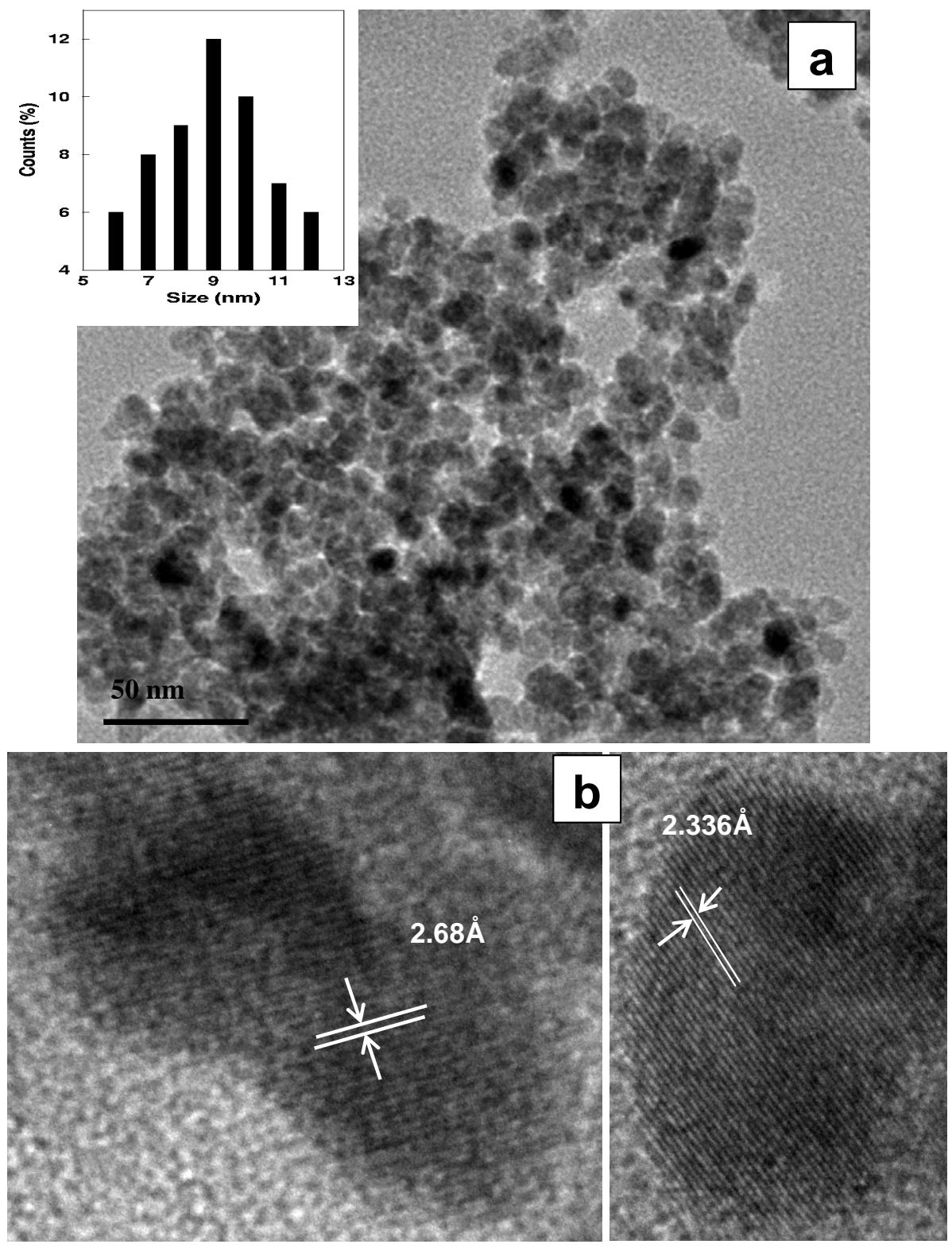


\section{Figure 3}
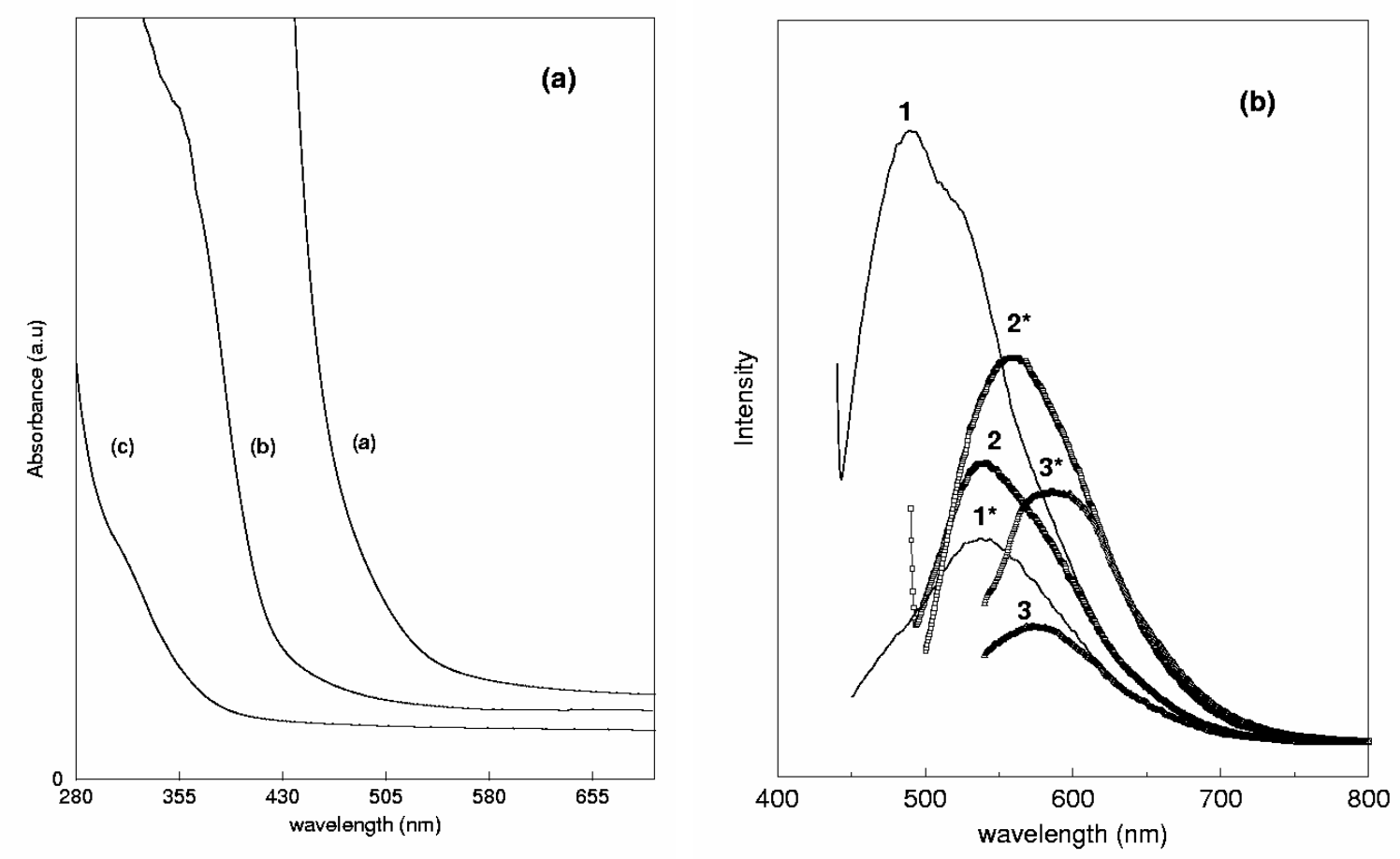
Figure 4

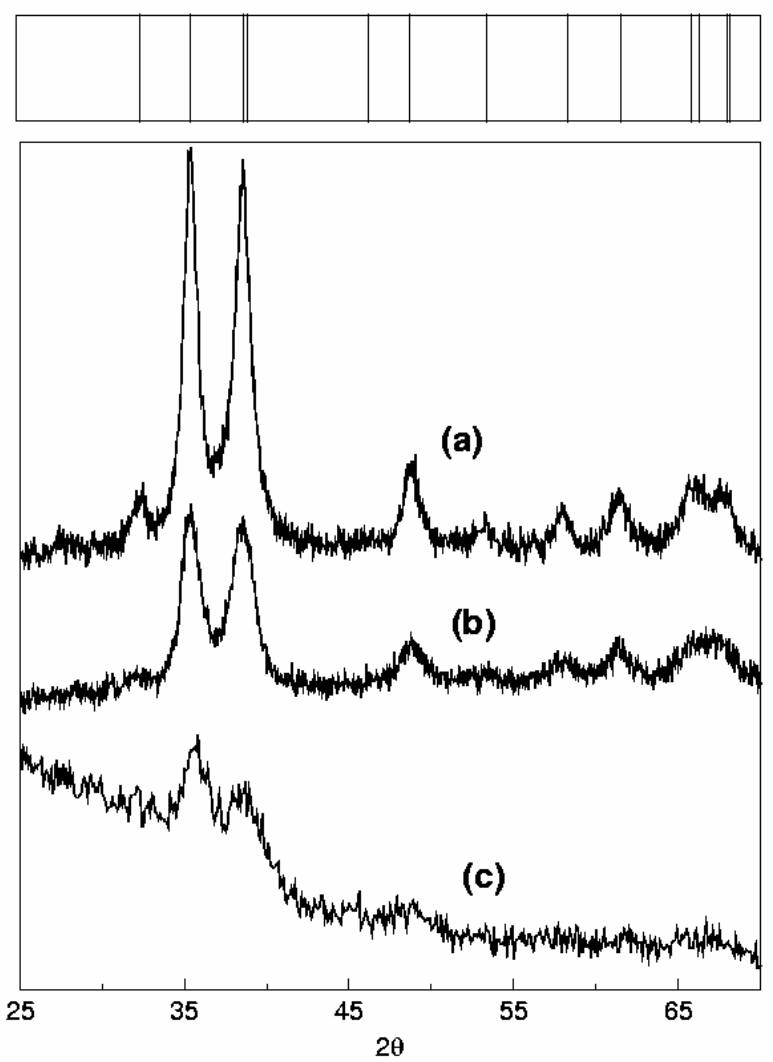


Figure 5
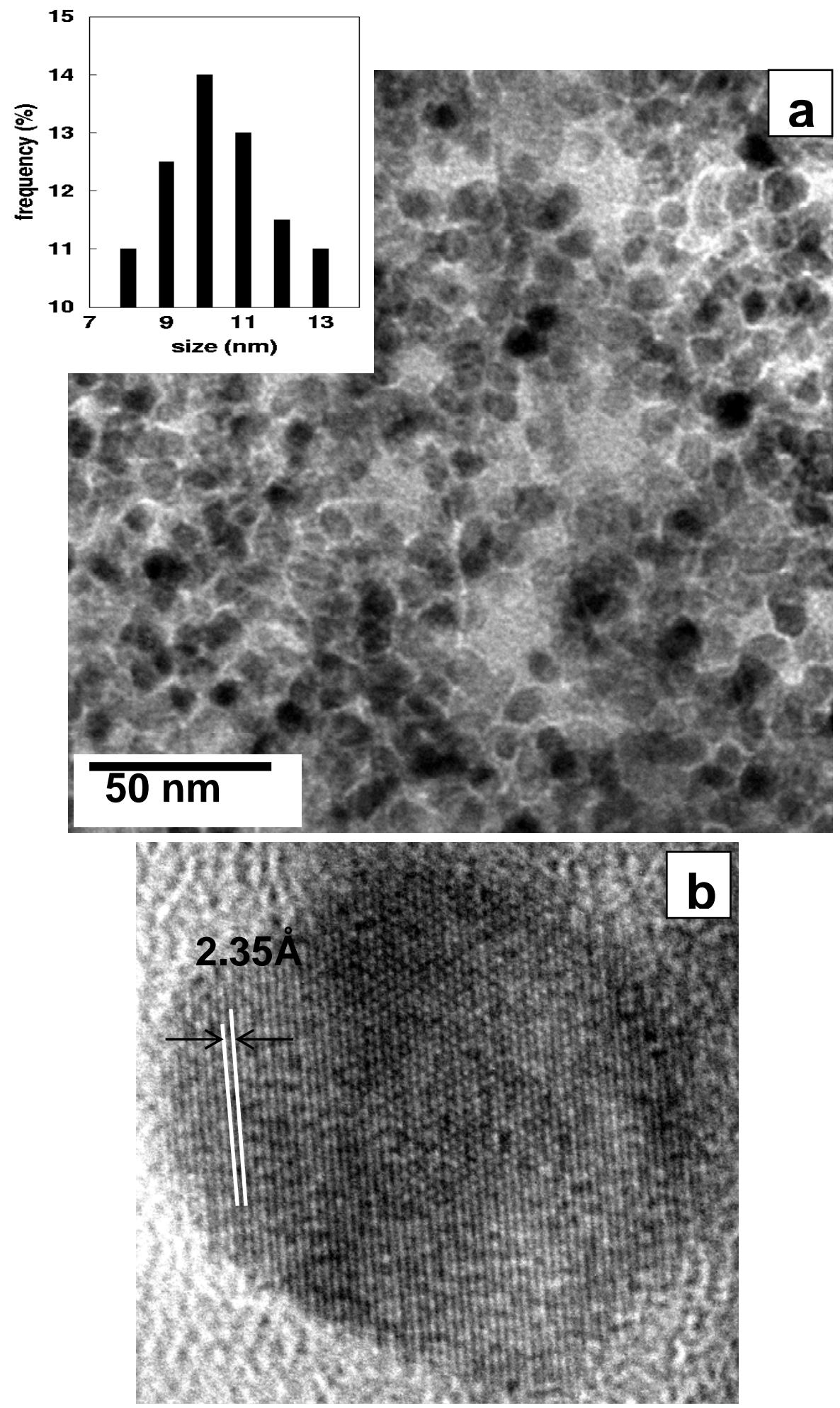